\title{
Limatus martiali Senevet et Abonnenc, 1939 et Wyeomyia nigricepbala n. sp.

\author{
(Diptera, Culicidae) \\ de la Guyane française
}

par J. CLASTRIER et J. CLAUSTRE

Muséum national d'Histoire naturelle, Entomologie

45, rue Buffon, F 75005 Paris.

Institut Pasteur de la Guyane Française, B.P. 304, 97305 Cayenne.

\section{Résumé.}

Redescription de Limatus martiali Senevet et Abonnenc, 1939 ( $\delta$ ), et description de Wyeomyia nigricephala n. sp. ( $\hat{\jmath})$, d'après des spécimens capturés en Guyane Française.

\section{Summary.}

Limatus martiali Senevet and Abonnene, 1939 and Wyeomyia nigricephala n. sp. (Diptera, Culicidae) from French Guiana.

The authors give a redescription of Limatus martiali Senevet and Abonnenc, 1939 ( $\hat{\text { ) }})$ and describe Wyeomyia nigricephala n. sp. ( $\delta$ ), from specimens collected in French Guiana.

Dans un lot de Moustiques capturés en Guyane Française se trouvaient deux mâles de la tribu des Sabethini, qui sont étudiés ci-dessous. L'un revient à une espèce déjà connue mais rarement rencontrée, Limatus martiali Senevet et Abonnenc, 1939 ; l'autre représente une espèce nouvelle, décrite sous le nom de Wyeomyia nigricephala.

Accepté le 10 mai 1978.

Annales de Parasitologie humaine et comparée (Paris), t. 53, $\mathrm{n}^{\circ} 5$. 


\section{Limatus martiali SenEVet et ABonNenc, 1939.}

Un mâle, capturé dans la forêt du Gallion le 3 septembre 1977 en fin de matinée, nous a incités à revoir l'holotype et le paratype de la collection G. Senevet (conservée au Laboratoire d'Entomologie du Muséum national d'Histoire naturelle), et à donner une nouvelle description, élargie, de cette forme adulte. La publication originale est en effet limitée à la seule pince génitale, elle n'a jamais été reprise ni complétée, et appelle certaines rectifications.

\section{MÂLE.}

Vertex et occiput entièrement couverts de larges écailles appliquées noires, à reflets bleutés; les mêmes écailles se retrouvent sur les faces latérales de la tête où elles sont plus ternes. Antenne $(\mathrm{L}: 1,40 \mathrm{~mm})$ à peine un peu plus longue que la trompe ; entièrement noirâtre. Clypeus également noirâtre. Palpe caché, invisible. Trompe (L : 1,36 mm) bien plus courte que le fémur antérieur $(0,66)$; régulièrement cylindrique, à peine recourbée ventralement et grossie à son extrémité ; noire à reflets cuivrés, avec une étroite bande ventrale d'écailles iridescentes bleutées sur sa moitié distale, à l'exception de l'apex qui est noir.

Mésonotum comparable à celui de L. durhami Theobald, 1901, c'est-à-dire présentant 5 larges bandes d'écailles appliquées d'un jaune doré ; 3 dirigées en avant (l'une axiale, chacune des 2 autres obliquement dirigée vers l'épaule correspondante) et 2 postérieures (obliquement dirigées vers l'angle postéro-latéral). Ces bandes, coalescentes dans la partie centrale du mésonotum, tranchent vivement sur le fond d'écailles noires. Des soies très courtes et dorées sur le bord antérieur; très longues et noires dans la région supra-alaire. Scutellum couvert d'écailles noirâtres. Post-scutellum à tégument jaune brunâtre; légèrement caréné ; sans écailles; portant dans sa partie postérieure une touffe de 5 à 6 soies jaune doré. Pronotum antérieur bien développé ; écailles roussâtres dorsalement; jaunes à reflets dorés ventralement ; une dizaine de soies brunes sur son bord distal. Pronotum postérieur semblable au précédent à la base ; s'assombrissant dorsalement. Pleures: tégument jaune brunâtre dorsalement; progressivement assombri ventralement ; couvert d'écailles incolores à reflets dorés, sauf sur la sternopleure inférieure et le métapleuron qui sont nus. Chétotaxie: pas de soies préstigmatiques, mais des écailles larges, noirâtres ; 2 longues soies propleurales ; 2 préalaires ; 3 à 4 mésépimérales supérieures.

Aile (L. : $2,30 \mathrm{~mm}$ ). Costa noire à son extrême base; progressivement éclaircie distalement, jusqu'à prendre une teinte grisâtre, comme les autres nervures et la frange. Balancier à tige jaunâtre et bouton brun-noirâtre.

Pattes. Hanches couvertes d'écailles sombres à reflets blanchâtres sur la paire antérieure; d'écailles plus claires que les précédentes et sans reflets sur les deux autres 
paires; partout, de longs poils dorés mêlés à ces écailles. Trochanters jaunâtres. Les articles suivants d'un noir assez terne; marqués de plages claires de la façon suivante. Fémur antérieur jaune doré sur le cinquième proximal. Fémur intermédiaire blanchâtre à son extrême base; les 3 derniers tarsomères correspondants entièrement d'un blanc pur argenté sur leur face ventrale. Sur la paire postérieure, une bande ventrale blanchâtre s'étendant sur toute la longueur du fémur, du tibia, et du basitarse. Griffes courtes et simples sur les pattes pro et métathoraciques, et les articles qui les portent normaux, cylindriques. Le $5^{\circ}$ tarsomère mésothoracique est au contraire globuleux sur plus de sa moitié proximale, cylindrique sur le reste; l'une des griffes est de la forme habituelle, simple; l'autre est beaucoup plus forte, plus longue et plus incurvée, elle présente à son extrémité une amorce de courbure inverse de la précédente, qui se termine par une longue épine rectiligne. Dimensions des fémurs, tibias et tarsomères en microns;
P. antérieure
2050
1950
1100
850
600
325140
P. intermédiaire
$2450 \quad 1725$
950
850
620
280100
P. postérieure
1500
1500
1950
1100
$800 \quad 300 \quad 150$

Abdomen. Sur le tergite I, un alignement transversal bien fourni d'écailles et soies d'un jaune doré. Les suivants (II-IV) noirâtres à reflets bleutés dans leur partie médiane, d'un brun noirâtre terne latéralement. Sternites en grande partie cachés; ce qui est visible, brunâtre.

Pince génitale (fig. 1). Nous n'apporterons qu'un complément d'informations à la description de Senevet et Abonnenc, et quelques rectifications. Plaque interne du coxite (A, pi) portant une soie ventrale longue et vigoureuse, accompagnée de 7 autres soies plus courtes et plus grêles. Dististyle court, trapu, légèrement recourbé en $\mathrm{S}$ très ouvert. $\mathrm{La}$ courbure proximale, à peine rétrécie depuis la base, n'offre aucune particularité. La courbure distale, qui ne compte qu'un seul lobe allongé, est au contraire assez complexe. Son bord dorsal, convexe et nu, se termine par l' «épine apicale» de Senevet et Abonnenc (a), qui est munie d'une petite soie. La partie médiane, saillante $(b)$, porte de 6 à 7 soies vigoureuses sur son arête ( 2 à 3 proximales courtes, 4 distales longues), et 4 autres beaucoup plus faibles à la base, ( 2 proximales groupées, 2 distales largement séparées). Sur le bord ventral on observe successivement, depuis la base, 4 formations différentes. D'abord, l' « appendice rétrograde » $(c)$ de la publication originale, légèrement encoché sur son bord distal. Ensuite, une touffe de soies falciformes à très long pédicelle $(d)$, paraissant réparties sur 2 plans, au nombre de 5 à 6 par plan (un seul est représenté sur la figure), plus une feuille triangulaire, également à long pédicelle $(e)$, située au-dessous des précédentes. Cet ensemble, dont la disposition régulière a été altérée par le montage sur le type et le paratype, correspond à la «dizaine de soies fines, recourbées, dont les plus apicales sont assez longues, et les plus voisines de la base courtes... ». Les 10 éléments suivants $(f)$ méritent une mention particulière. Ils sont représentés par Sene- 
vet et Abonnenc sous la forme de boudins allongés sur l'un de leurs schémas, et sous la forme de longues dents acérées sur l'autre. Sur le spécimen examiné (A, $f)$, ils ont l'aspect de soies longues, légèrement incurvées, réparties en deux groupes se faisant face par leur concavité (les 2 éléments médians manquent). Aussi est-ce avec surprise que sur le type et le paratype nous avons découvert de véritables feuilles, régulièrement et symétriquement grossies depuis la base, puis brusquement rétrécies à leur extrémité; les plus longues étant situées au centre de la série, et les plus courtes à chacune des extrémités. Ce sont les propres feuilles du type qui ont été rapportées sur le schéma $\mathrm{B}, f$, afin d'en donner une représentation réelle, sinon perceptíble sur les préparations récentes. Il semble, en effet, que ce changement d'aspect dans le temps soit dû à la dessiccation du milieu de montage, et à son amincissement consécutif. Les feuilles, normalement situées dans différents plans perpendiculaires à celui de la lame porte-objet, ont été amenées à pivoter de $90^{\circ}$ sur leur axe, et à se placer

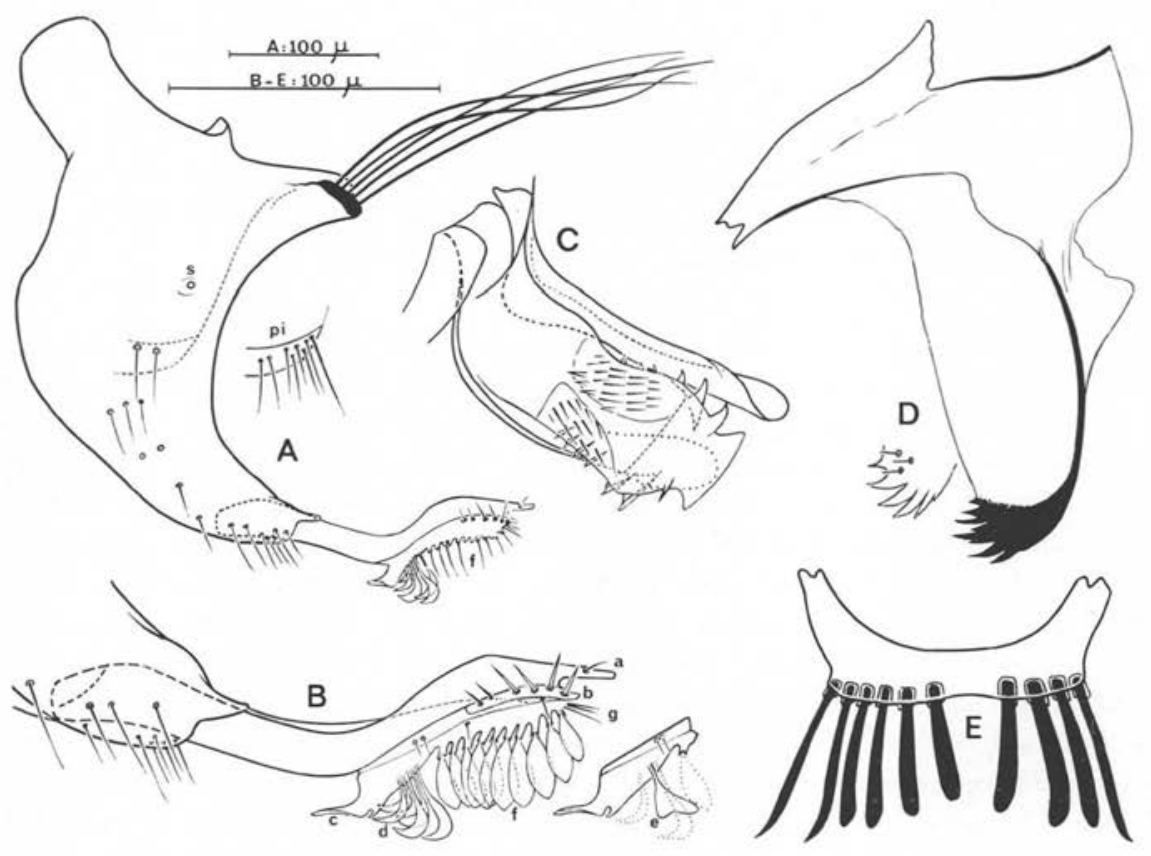

Fig. 1. Pince génitale de Limatus martiali Senevet et Abonnenc, 1939, o. A : coxite et dististyle vus par leur face externe $f$ : grandes feuilles du dististyle, telles qu'elles se présentent sur le spécimen récemment capturé ; pi : plaque interne du coxite; $s:$ base d'implantation de la grande soie solitaire). B : dististyle approximativement grossi deux fois (pour les lettres $a-g$, voir le texte). C: mésosome vu par la face ventrale. D: $\mathrm{X}^{\mathrm{e}}$ sternite dont l'extrémité est représentée sur ses deux faces. E: IX ${ }^{e}$ tergite. Figure exécutée d'après le spécimen récemment capturé, à l'exception des grandes feuilles $\mathrm{B}, f$, qui sont reproduites d'après l'holotype. 
dans un même plan, parallèle à celui du support. Ces feuilles, avec les soies portées par la partie médiane $b$, paraissent correspondre aux \& autres soies de cette partie apicale, disposées sur deux ou trois rangs, (qui) sont parfois un peu épaisses et légèrement recourbées », de la publication originale. La dernière formation du bord ventral consiste en 3 petites soies rigides $(g)$, dont les rapports avec le dististyle sont difficiles à préciser. Peut-être s'agit-il de feuilles semblables à celles de la série $f$, n'ayant pas pivoté sur leur axe en raison de leur très petite taille. Le mesosome (C) porte de chaque côté des alignements de petites crêtes triangulaires, dressées à angle droit sur le tégument, d'autant plus développées qu'elles sont plus distales, dont l'aspect varie considérablement suivant leur orientation, c'est-à-dire leur localisation. Vues de chant sur la partie ventrale du mesosome, elles sont semblables à des caractères cuneiformes noirâtres; vues de profil latéralement, elles apparaissent claires et plus ou moins recourbées en crochets. $\mathrm{IX}^{\bullet}$ tergite $(\mathrm{E})$ et $\mathrm{X}^{\bullet}$ sternite $(\mathrm{D})$ conformes à la publication originale.

L. martiali n'a pas encore été signalé hors du territoire de la Guyane Française. Le genitalia représenté sous ce nom par Lane (1953) est très différent de celui qui vient d'être décrit, et doit vraisemblablement être rapporté à une autre espèce.

\section{Wyeomyia nigricepbala n. sp.}

MÂLE.

Vertex et occiput entièrement couverts de larges écailles appliquées noires, à reflets légèrement bleutés ou dorés, à l'exclusion de toute écaille blanche. Antenne (L : 1,60 mm) presque aussi longue que la trompe ; torus noir dorsalement, brun noirâtre ventralement; flagelle noir. Clypeus d'un jaune brun assez clair; portant à son extrémité de minuscules écailles jaune pâle à reflets blancs. Trompe (L: 1,65 mm) nettement plus courte $(0,82)$ que le fémur antérieur; coudée ventralement à $1 \mathrm{~mm}$ de la base ; la partie proximale étroite et cylindrique ; la partie distale brusquement et fortement grossie dans le sens dorso-ventral; entièrement noire. Palpe dépassant le clypeus de la longueur de son dernier article; noir.

Mésonotum à tégument brun sombre; couvert d'écailles semblables à celles du vertex mais plus ternes; une très petite plage d'écailles blanches dans sa partie antérieure et médiane. Soies antérieures et supra-alaires claires, à reflets dorés ; une large touffe postéro-latérale d'écailles et soies noirâtres; pas de soies préscutellaires vues. Scutellum : tégument brun noirâtre basalement, plus clair distalement ; écailles noirâtres partout. Post-scutellum brun noirâtre; tégument grenu, présentant une ébauche de carène; pas d'écailles; une touffe postérieure de 8 soies brun clair. Pronotum antérieur bien développé ; couvert d'écailles semblables à celles du vertex dorsalement, d'écailles brun clair basalement ; 6 à 7 longues soies noires sur le bord dorsal. Pronotum postérieur: écailles appliquées noirâtres dorsalement; écailles du même brun clair que sur le pronotum antérieur basalement. Pleures brun noirâtre dans leur ensem- 
ble, et entièrement couvertes d'écailles transparentes claires à reflets blanchâtres, à l'exception du métapleuron, qui est jaunâtre clair, et nu. Chétotaxie : toutes les soies claires à reflets dorés ; 2 propleurales ; 2 préstigmatiques; 2 préalaires; 3 sternopleurales inférieures; 7 à 8 mésépimérales supérieures formant une longue touffe dressée.

Aile (L: 2,60 mm). Costa noire à reflets bleutés; les autres nervures et la frange légèrement enfumées; extrême base de l'aile jaunâtre postérieurement, comme le métapleuron. Toutes les écailles larges, sauf sur les deux branches de la $5^{\circ}$ nervure longitudinale $(\mathrm{Cu} 1, \mathrm{Cu} 2)$ où elles sont longues et étroites; 5 soies très longues sur l'alula, 1 seule vue sur la squamula. Balancier: moitié basale de la tige du même jaune léger que le métapleuron; moitié distale et bouton noirs.

Pattes: tégument des hanches de la même teinte que les pleures basalement, plus clair distalement ainsi que sur les trochanters. Des écailles semblables à celles des pleures, plus des soies claires, sur les premières. Les articles suivants noirs à reflets cuivrés, marqués d'écailles blanches de la façon suivante. Sur le fémur antérieur, une étroite bande ventrale n'atteignant pas l'apex, suivie de quelques écailles à la base du tibia. Sur toute la longueur du fémur et du tibia intermédiaires, une bande ventrale progressivement rétrécie depuis la base ; quelques écailles grisâtres sur le basitarse correspondant. Le fémur postérieur est semblable au précédent; sur le tibia, une bande moins large et moins marquée ; quelques écailles grisâtres éparses sur le basitarse ; face ventrale du $4^{\circ}$ tarsomère sauf l'apex, et du $5^{\circ}$ sur toute sa hauteur, d'un blanc pur. Griffes simples, égales ou subégales sur la même paire, mesurant respectivement 35 , 40 et 32 microns environ. Sur la paire mésothoracique, l'une d'elles est fortement grossie, à peine recourbée en $\mathrm{S}$ très ouvert, cylindrique, et à extrémité arrondie. Dimensions des fémurs, tibias et tarsomères en microns :
P. antérieure
$2000 \quad 1800$
1080
500
460
300
P. intermédiaire
1950
1550
1150
$\begin{array}{lll}780 & 520 & 280\end{array}$
P. postérieure
1600
1600
1850
1080
920740

Abdomen. Sur le tergite $\mathrm{I}$, une rangée basale et transversale de longs poils brun clair à reflets dorés, le reste comme sur les segments suivants. Ceux-ci (II-VI) entièrement couverts d'écailles appliquées à reflets ni bleutés ni cuivrés, mais plutôt d'un gris pruineux ; leur teinte, de noirâtre à noire dans la partie médiane du tergite, s'atténue latéralement; en même temps, ces écailles se mêlent progressivement à celles des sternites, de telle sorte que la ligne de séparation, quoique rectiligne, reste assez imprécise. Sternites entièrement jaunâtres, avec quelques soies dorées sur leur bord postérieur.

Pince génitale (fig. 2). Coxite (A) étroit et allongé; modérément coudé dorsalement sur son dernier quart. Sur la face interne, les 3 soies dorsales, longues, fortes, 
amplement recourbées, plaque interne portant une quinzaine de soies courtes et grêles, toutes semblables (il n'a pas été vu de soie plus longue, mais il n'est pas possible d'assurer qu'elle n'est pas perdue). Sur la face externe ; une étroite bande médiane et transversale d'écailles allongées (3 d'entre elles sont représentées sur la figure), suivie distalement par une pilosité clairsemée, courte et raide ; la longue soie isolée $(s)$ perdue ; tégument pigmenté sur les parties couvertes.

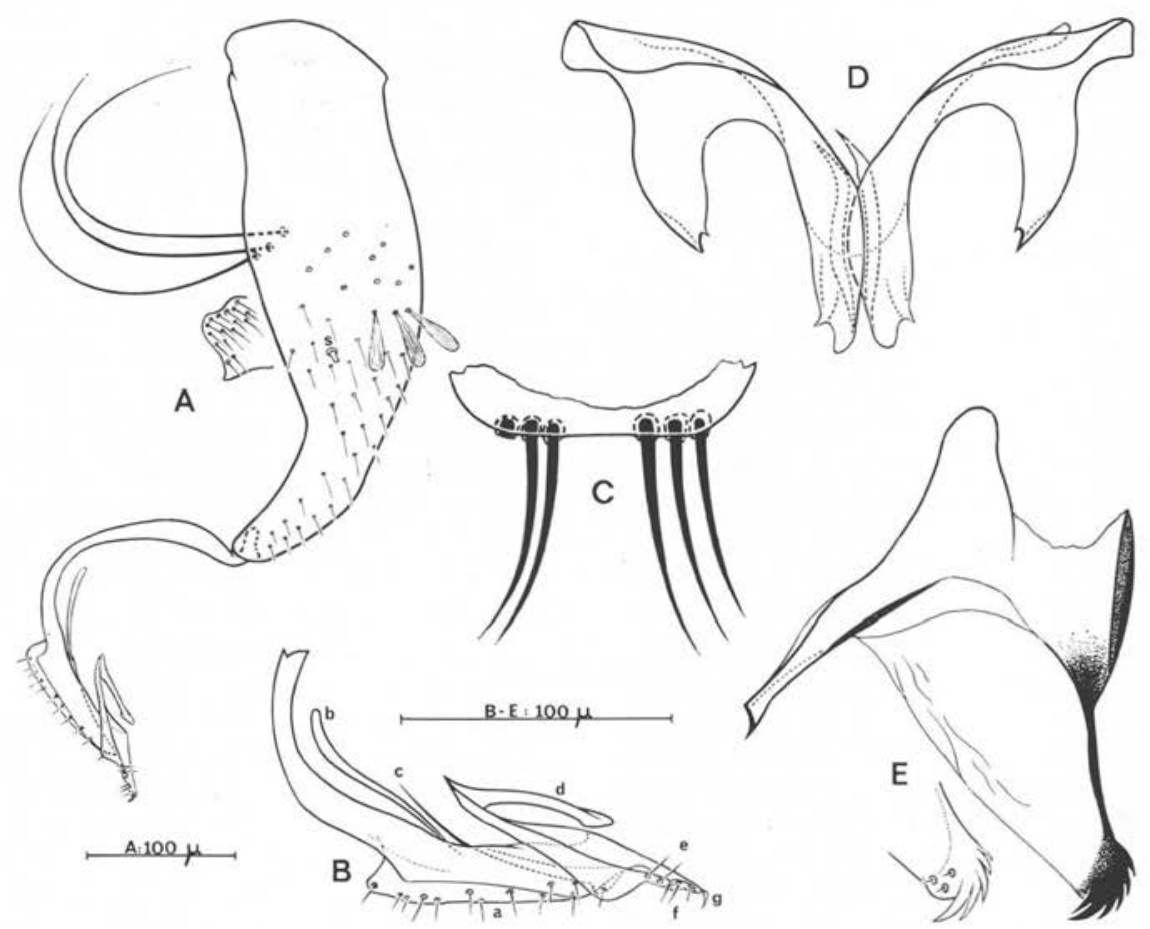

Fig. 2. Pince génitale de Wyeomyia nigricephala n. sp., ô. A : coxite et dististyle vus par leur face externe ( $s$ : base d'implantation de la grande soie solitaire). B : extrémité du dististyle approximativement grossie deux fois (pour les lettres $a-g$, voir le texte). C: IXe tergite. D : mésosome vu par la face ventrale. E: $\mathrm{X}^{\circ}$ sternite dont l'extrémité est représentée sur ses deux faces.

Dististyle (A) presque aussi long que le coxite, mais paraissant de moitié plus court en raison de la très forte courbure de sa partie proximale. La partie distale (B), allongée et modérément grossie, porte sur son bord dorsal une douzaine de soies courtes et raides $(a)$. Sur le bord ventral, on observe successivement depuis la base: une feuille étroite et très allongée $(b)$; un filament (ou feuille ?) mal défini $(c)$; un appendice latéral en forme de crochet $(d)$ dont l'ouverture est orientée vers l'apex ; un groupe de 2 soies dirigées ventralement $(e)$; un autre groupe de 3 soies plus faibles dirigées torsalement $(f)$; un appendice terminal $(g)$ présentant l'aspect d'un bec ou d'une feuille 
arrondie suivant son orientation. $\mathrm{VIII}^{\circ}$ segment perdu. $\mathrm{IX}^{e}$ tergite $(\mathrm{C})$ très étroit, rectiligne, à lobes inapparents ; portant deux groupes largement séparés de 3 épines d'autant plus longues que plus internes, fortes, à extrémité acérée, s'écartant du plan de symétrie de la pince. Extrémité du $\mathrm{X}^{\circ}$ sternite $(\mathrm{E})$ armée de 5 à 6 dents bien découpées, et portant 3 soies très courtes. Mesosome (D) représenté sur la figure.

Guyane Française. Forêt du Gallion, 3 septembre 1977, capturé au filet en fin de matinée : 1 ơ, holotype, déposé au Muséum national d'Histoire naturelle.

Parmi les espèces dont le mâle est connu, il en est très peu qui possèdent sur le bord ventral du dististyle un appendice en forme de crochet ouvert vers l'apex; chez ces espèces, la partie proximale du dististyle est rectiligne, courte et forte, trapue ; en outre, la morphologie de la partie distale est tout à fait différente, et ne peut prêter à confusion.

Si l'on se réfère à Lane (1953), l'absence d'écailles céphaliques blanches sépare $W$. nigricephala de toutes les espèces connues par la seule femelle, qui portent de telles écailles sur deux au moins des localisations suivantes: vertex, marge oculaire, mentum. En outre, ces mêmes espèces possèdent l'une au moins des caractéristiques suivantes, opposées à celles de l'espèce nouvelle: écailles blanches sur le pronotum antérieur ; séparation des teintes de l'abdomen se faisant suivant une ligne en dents de scie ou profondément ondulée; squamula portant 2 soies ou sans soie ; tarse postérieur entièrement sombre.

\section{Bibliographie}

Lane J. (1953) : Neotropical Culicidae, T. 2, 551-1112, Univ. of São Paulo, édit., São Paulo.

Senevet G., Abonnenc E. (1939): Les Moustiques de la Guyane Française. III. Les Sabéthinés. Arch. Inst. Pasteur Alger, 17, 247-281. 